\title{
Survey on Energy Efficient Cloud: A Novel Approach towards Green Computing
}

\author{
${ }^{* 1}$ Anup Gade, ${ }^{2}$ Nirupama Bhat, ${ }^{3}$ Nita Thakare \\ 1,2 Vignan's University, Guntur, AP, \\ ${ }^{3}$ Priyadarshani College of Engineering, Nagpur, MS \\ *Email: gadeanup@gmail.com
}

\section{Received: 09 ${ }^{\text {th }}$ July 2018, Accepted: $14^{\text {th }}$ August 2018, Published: $31^{\text {st }}$ August 2018}

\begin{abstract}
Cloud computing is acting at leading role in world's technical industry but at the same time it is contributing around $02 \%$ in pollution by emitting $\mathrm{CO} 2$. It's probably the most appropriate moment to touch this sensitive area of discussion as global warming and Paris act is a hot cake in the market. The idle terminal in data centers and heat dissipated by these devices makes significant contribution in making atmospheric changes. There is number of algorithms available through which effective load balancing and power saving is possible. Migration of Virtual Machines (VM) so as to reduce requirement of physical machines is taken into under consideration so that overall power consumption will also be reduced. Energy consolidation is also an area where light need to be thrown. It's time to implement a combinatorial approach where effective changes at server level and client level can make the significant difference. Developing green cloud is need of an era to avoid global carbon foot print.
\end{abstract}

Keywords: Green Cloud, Migration of VM, Load Balancing, Data Centers.

\section{Introduction}

In recent business world Cloud Computing is a facility which an organization need not have to own, it's kind of provision that you can use on rent basis as per organization's requirement. This concept of cloud makes it more popular and companion to the firms which are not willing or not in a state (small firms) to invest funds in three basic areas where cloud services are available on hire basis. Providing services on rent basis is one of the reason behind popularity of cloud and others are its capacity of storing chunks of data may be Terabytes a day, its ability to provide Quality of Service (QoS), Maintaining the service terms we call it Service Level Agreement (SLA), ease of availability in a single click you can hire it, need not have to worry about its maintenance, etc. Cloud consist of three basic service models at the bottom it lies hardware part generally call it as Hardware as a Service (HaaS) or Infrastructure as a Service (IaaS) this layer consist of all hardware required including databases, servers networks devices, etc. Second layer from bottom is Platform as a Service (PaaS) it will provides us the platform for implementation and operation on cloud multiple platforms are available like Xen, VM, Nebula, etc. The top layer is Software as a Service (SaaS) all development tasks are done at this layer. The best examples are Google drive, Facebook, Salesforce.com, etc. All these three layers proved its utility and market has accepted the concept of cloud unanimously but there are certain areas where researchers have concerned as, energy consumed by a single data center, minimizing the number of idle machines in data centers, security issues, minimization of SLA violation and converting cloud into smart cloud.

In next sections of article we will see the related works in the area of green cloud further we will see the comparative analysis of various articles. The subsequent sections will cover the discussion part and proposed system to make the cloud echofriendly.

\section{Related Work}

Energy consumed by data center has attracted attention of many researcher and consistent efforts of research community is going on to convert cloud into green cloud. In [1], authors proposed improved version of clonal selection algorithm (ICSA) which proved to be better in the area of response time and has taken minimal time for task execution. The algorithm contributed in reducing make span by giving affinity function. ICSA uses concept of mutation in which weak antibodies are replaced with best one from the available. System will run ICSA every time whenever new request will arrive to justify the allocation. The algorithm proved its betterment than IDEA, EMLS and DVS approach in terms of response time, task completion and data center's efficiency.

Chao-Tung Yang et al. [2] given a technique which effectively handles the power of a green cloud on the basis of formula derived using gross occupied ratio, the formula suggest us that if ratio is more than the threshold value then additional physical machine needs to be added else one or more number of physical machine can be made free using live migration and can be shutdown to save the power. The process gain considerable results but considered only two factors CPU utilized and Memory used to get better and more precise results disk space and network bandwidth are factors need to take into consideration. 
Anton Beloglazov et al. [3] have addressed multiple issues which mainly leads cloud into energy-aware cloud. They proposed green cloud architecture consisting of Consumers benefited by cloud services, Service Allocator its responsibility is to finalize negotiation of SLA according to Consumer's service requirement, Analyzer need to have prior information about resource requirements, Energy Monitor its main task is to supervise the allocation of resource and provide feedback to VM manager to finalize energy aware resource allocation, Scheduler will decide which VM to remove and to add without disturbing the quality of services. The prior intention is to use VMs at minimal performance level or it can be turned off unused VMs. They have worked for allocating resources in consideration with its energy consumption by modifying VM Placement algorithm popularly named as Best Fit Decreasing algorithm. The VM selection is based on two steps in first step the probable VM which is supposed to shift is chosen and in second step using Modified Best Fit Decreasing (MBFD) algorithm the selected VM will placed on host. It decides upper and lower limit of threshold, if host's utilization is close to lower bound VMs associated with this host need to migrate and host has to switched off or take into sleep mode this will help in reducing power consumption. The live migration strategy is used to avoid violation of SLA. They have proposed, Minimum Migration (MM) the policy which reduces the frequency of migration of virtual machines, Highest Potential Growth policy (HPG) under which the VMs in which CPU is underutilized is migrated and Random Choice (RC) policy will select the VMs randomly to reduce the utilization of CPU below upper bound threshold.

Hwa Min Lee et al. [4] proposed resource allocation based on performance analysis for reduction in turnaround time of a job and minimizing the power utilization thereby achieving the effective utilization of VMs. Multiple LU decomposition methods are available (it is modified form of Gaussian elimination) like Crout, Doolittle and Cholesky in which they have Doolittle to show performance analysis of the algorithm. In this article optimal performer (node) is selected by CPU gives the rating to memory, storage and node. In resource allocation algorithm when any task is submitted to cloud for execution the task will be divided into several subtasks and then the scheduler will decide which subtask is executed by which cloud based on available information from all schedulers. This algorithm is used at hypervisor to execute the allocated task in minimum time and for effective utilization of resources.

Yingyou Wen, Zhi Li, et al. [5] summarizes their article by successfully implementing algorithm for VM placement which is ideally improved form of fundamental genetic algorithm. They have given method so that patterns of the physical machines
(PMs) load can be observed in association with the corresponding threshold of PMs. The PM is recommended by the algorithm which must be a part of pool and further they have proposed an algorithm which suggests us how to select VM based on the criteria of least migration strategy. Their VM placement algorithm is based on five factors as encoding where they have used First Fit Algorithm, fitness function for selecting best chromosome as parents, selection to create offspring, crossover to generate the best gene and finally mutation in which two randomly selected chromosomes are swapped. The iterations will stop whenever there will be improvement in the fitness of the chromosomes.

C. Zhu et al. [6] discussed Sensor-Cloud gaining interest of both academician and researchers as it contains the features of WSNs as sensing and collecting the data, being a sensory data on cloud users have the ability to access it from anywhere else. It can increase the lifetime of sensor by $3.25 \%$ and reduced power utilization by $36.80 \%$ which is really important for smart world. They have given multiple suggestions through which energy efficiency can take place 1) Installation of nonconventional energy sources (wind, solar energy, water, etc.); 2) Taking help from dynamic-power management techniques (e.g. vSphere); 3) Design data-centres consuming less power as nano data centers is a novel idea to minimize power requirements; 4) Developing energy-aware routing techniques to maintain the flow of network and keep useless devices in sleep mode.

Areas to work on while dealing with green cloud computing are 1) Placement of Virtual Machines across multiple resources. 2) For managing green power of virtual machine cluster factors like disk space and network bandwidth need to be considered. 3) Computational complexities like demand forecasting, calculation of overall threshold need to be considered. 4) Use of VM at minimum performance level. 5) In case of using algorithms having iterations then number iterations to follow is a matter of concerned.

\section{Comparative Analysis}

We have identified six basic things which green cloud computing should focus as VM migration policies, energy saving, minimizing task completion time, SLA violation percentage and highest potential growth policy which focuses on maximizing CPU utilization. Table 1 depicts the picture showing relevance of literature with above mentioned key areas.

\begin{tabular}{|c|c|c|c|c|c|}
\hline \multirow{2}{*}{$\begin{array}{c}\text { Survey } \\
\text { Focused on } \\
\text { key points }\end{array}$} & \multicolumn{6}{|c|}{ Survey } \\
\cline { 2 - 6 } & $\underline{[1]}$ & $\underline{[2]}$ & $\underline{[3]}$ & $\underline{[4]}$ & {$[\underline{[5]}$} \\
\hline $\begin{array}{c}\text { VM } \\
\text { Migration } \\
\text { Policies }\end{array}$ & & $\sqrt{ }$ & $\sqrt{ }$ & $\sqrt{ }$ & $\sqrt{ }$ \\
\hline Energy & $\sqrt{ }$ & & $\sqrt{ }$ & & $\sqrt{ }$
\end{tabular}




\begin{tabular}{|c|l|l|l|l|l|} 
Saving & & & & & \\
\hline $\begin{array}{c}\text { Minimization } \\
\text { in Task } \\
\text { Completion } \\
\text { Time }\end{array}$ & $\sqrt{ }$ & $\sqrt{ }$ & $\sqrt{ }$ & & \\
\hline $\begin{array}{c}\text { SLA } \\
\text { Violation }\end{array}$ & & & $\sqrt{ }$ & & $\sqrt{ }$ \\
\hline $\begin{array}{c}\text { Highest } \\
\text { Potential } \\
\text { Growth } \\
\text { Policy }\end{array}$ & & $\sqrt{ }$ & $\sqrt{ }$ & & \\
\hline
\end{tabular}

Table1: Focus of Literature Reviewed Based on Key Areas

VM migration policies is a factor which is focused by maximum researchers, energy saving and task completion time are second most concentrated areas where as SLA violation and CPU maximization are the areas of concerned.

\section{Discussion}

Green cloud is concentrated on few areas mainly reduction in energy usage (energy saving), maximizing resource utilization with minimum SLA violation. Use of non-conventional energy sources, implementation of dynamic-power management techniques can play crucial role in minimization of power consumption. Energy aware routing techniques and development of nano data centers can contribute in the development of smart world [6]. Use of computing system consuming less power or a system with small form factors will enable the computing echo friendly. Concept of green cloud can be implemented in two areas first at server end where issues are different like minimum migration policy of VM [3], utilizing CPU to its fullest extent [2] and utilizing minimum number of physical machines or taking them into sleep mode those which are underutilized [1]. The second area is at data center level where power consumption of cooling can be reduced by setting up of data center in the area where normal temperature is below $5^{\circ} \mathrm{C}$. Normally a data center invests $40 \%$ of its power in cooling servers and network devices [8]. Use of alternative power options like geothermal, solar power and wind turbines by creating nano data centers can reduced the burden [6]. Two possible solutions exist for controlling the use of energy software solution and hardware solution. Software solution consist of efficient programming, data structures using less space and distributed computing whereas hardware solution involved Dynamic Voltage Frequency Scaling (DVFS) technique and effective power management techniques $[7,9,10,11,12,13]$.

\section{Proposed System}

In order to reduce power consumption with minimum task completion time we need to have system which will work on both the areas as first at the server side where VM commissioning takes place and second at the software level where computing power can be significantly increased. Our proposed system will work on minimizing the power usage at the Data center [count of VM's Vs Energy], comparative analysis how many number of PMs we will be able to closed-[count of VM'scount of closed PM's], comparison of Execution Time at Respective VM-[Execution Time Vs Cores Utilized] and maximize CPU Utilization by using capabilities of Multi-Core. Figure 1 shows pictorial view of theoretically proposed system

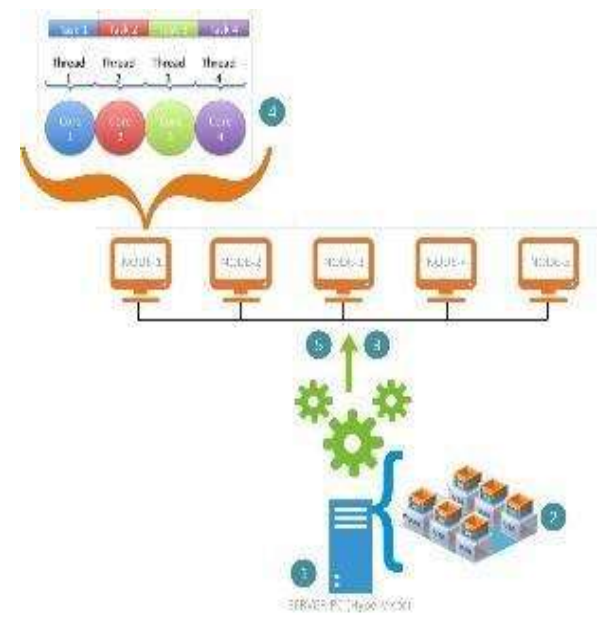

Figure 1: Pictorial View of Proposed System

Activities of the proposed system will work accordingly:

1. Load inputted to software at server computer.

2. Load values at software will be inputted to CloudSim simulator.

3. Simulation will compare improved genetic algorithm, improved clonal selection algorithm and proposed algorithm will produce the output in terms of node machine with execution time. The proposed algorithm is expected to provide least turnaround time than the existing algorithm.

4. Once the simulation has completed the output generated during simulation will be inputted to the software and respectively load will be distributed. This step will solve the issue of load balancing and thereby increase the efficiency of the system.

5. As load will be distributed by server to all node machines, they will perform desired task in parallel way by using multi-core programming.

6. Simulating load of 1000 images to be processed in CloudSim will generate 
tabular output of mean time with respect to individual machine.

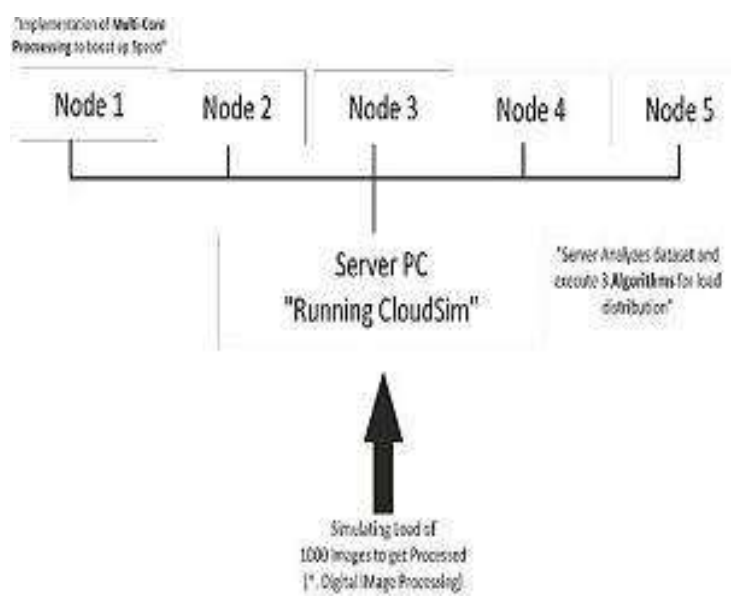

Figure 2: Activity Diagram of Proposed System

\section{Conclusion}

In this survey article we have compared some of the literatures based on six key areas of VM migration, energy saving, time required to complete allotted tasks, percentage of SLA violations and CPU utilization wherein it has been observed that still there is a significant scope for the improvement and development of the new system through which cloud computing can be truly converted into echofriendly computing. Our proposed work given in this article is currently in the developing stage out of which our proposed block diagram is given here through which our main intention is to reduced turnaround time, minimize number of physical machines in used, reduced response time and using the capabilities of multi-cores. The system with higher speed, better efficiency with minimum terminals it leads to the reduction in the overall power consumption which ultimately contributed in creating echo-friendly cloud.

\section{References}

1. Wanneng Shu, Wei Wang, Yunji Wang, "A novel energy-efficient resource allocation algorithm based on immune clonal optimization for green cloud computing", Springer Journal of Wireless Communications and Networking, Jan.-2014.

2. Chao-Tung Yang, Jung-Chun Liu, Kuan-Lung Huang, Fuu-Cheng Jiang, A method for managing green power of a virtual machine cluster in cloud, Elsevier- Future Generation Computer Systems 37 (2014) 26-36, March2014.

3. Anton Beloglazov, Jemal Abawajy, Rajkumar Buyya, "Energy-aware resource allocation heuristics for efficient management of data centers for cloud computing", Elsevier Future Generation Computer Systems, May-2011.

4. Hwa Min Lee, Young-Silk Jeong, Haeng Jin Jang, "Performance analysis based resource allocation fore green cloud computing", Springer Science + Business Media, New York, Sept.2013

5. Yingyou Wen, Zhi Li, et al., "Energy-Efficient Virtual Resource Dynamic Integration Method in Cloud Computing”, IEEE Access, 2017.

6. Chunsheng Zhu, Victor C.M. Leung, Lei Shu and Edith C.-H. Ngai, "Green Internet of Things for Smart World", IEEE Transaction, Special Section on Challenges for Smart Wolds, November 2015.

7. Laura-Diana Radu, "Green Cloud Computing: A Literature Survey", Symmetry, MDPI, November 2017.

8. Z. Songa, X. Zhangb, C. Erikssona, "Data Center Energy and Cost Saving Evaluation", Elsevier, Energy Procedia 75 (2015).

9. Kaur, G.; Midha, S.A., "Preemptive Priority Based Job Scheduling Algorithm in Green Cloud Computing", In Proceedings of the $6^{\text {th }}$ International Conference Cloud System and Big Data Engineering, Noida, India, 14-15 January 2016; IEEE: New York, NY, USA, 2016.

10.Wu, C.M.; Chang, R.S.; Chan, H.Y, "A green energy-efficient scheduling algorithm using the VFS technique for cloud data centers", Future Gener. Comput. Syst. 2014.

11.Procaccianti, G.; Lago, P.; Bevini, S, "A systematic literature review on energy efficiency in cloud software architectures", Sustain. Comput 2015.

12.Borah, A.D.; Muchahary, D.; Singh, S.K.; Borah, J, "Power Saving Strategies in Green Cloud Computing Systems", Int. J. Grid Distrib. Comput. 2015.

13.Carrega, A., "Repetto, M. Exploiting novel software development paradigms to increase the sustainability of data centers", In Proceedings of the 9th International Conference on Utility and Cloud Computing (UCC), Shanghai, China, December 2016; IEEE: New York, NY, USA, 2016 